\title{
Recombinant Ornithodoros moubata Saliva-derived Small Protein Complement C5 Inhibitor
}

National Cancer Institute

\section{Source}

National Cancer Institute. Recombinant Ornithodoros moubata Saliva-derived Small

Protein Complement C5 Inhibitor. NCI Thesaurus. Code C156154.

A recombinant small peptide inhibitor of the terminal complement pathway protein C5

originally derived from a protein in the saliva of the Ornithodoros moubata tick, with potential anti-inflammatory and cytoprotective activities. Upon administration, recombinant Ornithodoros moubata saliva-derived small protein complement C5 inhibitor targets and binds to a unique site on the terminal complement protein $\mathrm{C5}$, which blocks C5 cleavage into the pro-inflammatory components C5a and C5b, and prevents the C5bdependent assembly of the membrane-attack complex (MAC; C5b-9). This prevents MAC-mediated lysis and destruction of red blood cells (RBCs) in paroxysmal nocturnal hemoglobinuria ( $\mathrm{PNH}$ ) and prevents tissue destruction in various other complementmediated inflammatory and autoimmune diseases. In addition, this C5 inhibitor targets, binds to and inhibits the activity of leukotriene B4 (LTB4), thereby further preventing inflammatory-mediated cell destruction. 